\title{
La orientación psicopedagógica para la prevención de la dependencia tecnológica en los adolescentes del Preuniversitario
}

\section{The psych pedagogical orientation for the prevention of technological dependence in teenagers of the Pre-university}

\author{
Lic. Lixenda Abad Águila ${ }^{1}$ \\ lixenda@nauta.cu. \\ https://orcid.org/0000-0002-6195-337X \\ M. Sc. Leticia del Carmen Torres Zerquera ${ }^{2}$ \\ Ictorres@ucf.edu.cu ORCID \\ https://orcid.org/0000-0002-1593-7459 \\ Lic. Miguel Alejandro Pérez Egües 3 \\ mapegues@ucf.edu.cu \\ https://orcid.org/0000-0002-0861-7991 \\ Lic. Dayana Díaz Falcón ${ }^{4}$ \\ ddfalcon@ucf.edu.cu ORCID \\ https://orcid.org/0000-0001-8537-4025
}

\section{RESUMEN}

La orientación psicopedagógica en el Preuniversitario es un proceso que responde a las exigencias de la sociedad y a las necesidades propias de la etapa del desarrollo por la que transitan los educandos. En la actualidad, el uso desmedido e incontrolado de las tecnologías, constituye una de las problemáticas que requiere de la orientación psicopedagógica en la etapa de la adolescencia a fin de prevenir la dependencia tecnológica. El estudio efectuado en un Preuniversitario del municipio Cienfuegos develó insuficiencias en el proceso de orientación, que se manifiestan en la poca percepción del riesgo que entraña el uso desmedido de las tecnologías. El objetivo del trabajo consistió en proponer una estrategia de orientación psicopedagógica para la prevención de la dependencia tecnológica en los adolescentes del Preuniversitario; estructurada en cuatro etapas: diagnóstico, elaboración, ejecución y evaluación. Los resultados preliminares indicaron que es una estrategia de orientación psicopedagógica factible, que brinda a los adolescentes la posibilidad de ser protagonista de su perfeccionamiento y tiene en cuenta la participación de docentes y familiares.

\footnotetext{
${ }^{1}$ Psicopedagoga Preuniversitario Martín Dihigo Llano, Cienfuegos. Cuba.

2 Profesora Universidad de Cienfuegos "Carlos Rafael Rodríguez", Cienfuegos, Cuba.

3. Profesor Universidad de Cienfuegos "Carlos Rafael Rodríguez". Cienfuegos, Cuba.

4 Profesora Universidad de Cienfuegos "Carlos Rafael Rodríguez", Cienfuegos, Cuba.
} 


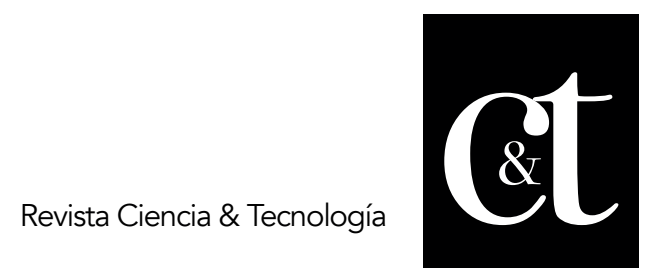

No. 28, 31 de octubre de 2020

ISSN impreso: 1390 - 6321

ISSN online: 2661 - 6734

Palabras clave: Adolescencia, dependencia tecnológica, orientación psicopedagógica, estrategia, Preuniversitario

\begin{abstract}
The psych pedagogical orientation in the Pre-university is a process that responds to the demands of society and to the needs of the stage of development through which the students pass. At present, the excessive and uncontrolled use of technologies constitutes one of the problems that requires psycho-pedagogical orientation in adolescence in order to prevent technological dependence. The study carried out in a high school in the Cienfuegos municipality revealed inadequacies in the orientation process, which was manifested in the low perception of the risk that the excessive use of technologies entails. The objective of the work consisted in proposing a psych pedagogical orientation strategy for the prevention of technological dependence in pre-university adolescents; structured in four stages: diagnosis, preparation, execution and evaluation. Preliminary results indicated that it is a feasible psych pedagogical orientation strategy, which offers adolescents the possibility of being the protagonist of their improvement and takes into account the participation of teachers and family members.
\end{abstract}

Keywords: Adolescence, technological dependence, psych pedagogical orientation, strategy, Pre-university

\title{
Introducción
}

La orientación psicopedagógica, concebida como un componente integral y favorecedor del proceso educativo, ha evolucionado desde su surgimiento hasta la actualidad. En este contexto la orientación se ha encaminado a satisfacer las necesidades de ayuda a los estudiantes para que movilicen de forma consciente sus potencialidades, enfrenten sus limitaciones y decidan el curso de su vida. Es así que debe ser vista como un proceso continuo, vital para todos los seres humanos, a fin de prepararlo para la realización eficiente de las "tareas del desarrollo" y prevenir así, la aparición de fallas o problemas en la formación de la personalidad.

La orientación se erige como un "proceso en el cual el sujeto es, ante todo, un ser humano que requiere un clima de confianza y de la seguridad física, psicológica y emocional. La calidad de las relaciones que se tienen con él, resulta un factor predominante en el aprendizaje, así como la motivación y la autoestima que también son factores claves en el aprendizaje y a su vez en el éxito del estudiante" (Rojas, 2018, p. 11).

Así mismo, dentro del proceso de orientación, se considera la prevención como uno de los principios básicos. Según Rodríguez (2001), la orientación supone actuaciones de tipo proactivo; se trata de actuar antes de que surja el problema. La prevención pone su énfasis en el grupo o comunidad, pretende reducir los riesgos de la totalidad de los miembros de un sistema, mejorando las condiciones existentes o previniendo posibles problemas. En tal sentido, el Ministerio de Educación de Cuba inserta la actividad preventiva en todas sus direcciones de trabajo y niveles de enseñanza. Es por ello que, aunque existe un conjunto de indicaciones generales sobre el trabajo preventivo, cada área de trabajo ha precisado una serie de orientaciones, principios y acciones educativas que garantizan el carácter preventivo de su labor (Del Pino y 


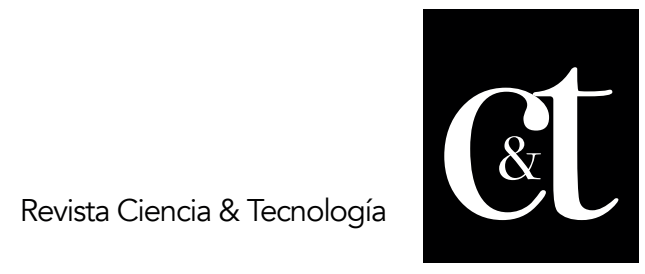

No. 28, 31 de octubre de 2020

ISSN impreso: 1390 - 6321

ISSN online: 2661 - 6734

Recarey, 2005). Así lo concibe la Resolución No.111/2017 en su objetivo general, el cual apunta a dotar a funcionarios, directivos de los diferentes niveles de dirección de la Educación Preuniversitaria, docentes, educandos y familias, de herramientas, formas y vías de trabajo para ejecutar el trabajo preventivo que asegure calidad en la formación integral de los adolescentes, basada en la equidad e igualdad de oportunidades y que les permitan ejecutar las acciones desde prácticas educativas inclusivas.

Es así que este nivel de enseñanza contempla las problemáticas y temas específicos generados por diferentes factores que requieren especial atención. Ello hace que cada contexto e institución educativa en particular despliegue acciones y actividades preventivas a modo de solucionar y/o atenuar las problemáticas existentes y evitar complicaciones futuras. En este sentido una de las problemáticas actuales que requiere de especial atención en el Preuniversitario está vinculada con el uso de las tecnologías. En la actualidad el desarrollo alcanzado por las tecnologías genera diversas preocupaciones a los sistemas educativos y otras agencias de socialización con las que interactúan los estudiantes. Su aparición ha provocado un cambio notable en la manera de trabajar, de relacionarse entre individuos, en la vida cotidiana y hasta en las actividades de ocio.

Es por ello que el plan de estudio provisional de Preuniversitario en el III Perfeccionamiento educacional refiere que los educandos pueden acceder a diferentes fuentes de información más allá de las promovidas por la institución educacional, en especial mediante internet y las nuevas tecnologías de la información y las comunicaciones (TIC), eso les lleva a realizar actividades que ocupan su tiempo pero amplían su visión de la sociedad y el mundo (Díaz, 2017).

Sin duda, las TIC contribuyen a aumentar la calidad de vida, facilitan gestiones o interacciones a distancia, reducen el tiempo empleado en desplazamientos y ofrecen más opciones de entretenimiento. Internet, los teléfonos móviles y los juegos interactivos tienen beneficios que han contribuido a su rápida implementación en la vida cotidiana, pero hacer un uso inadecuado de ellos implica riesgos para los más jóvenes (Encinas, Requesens y Helguera, 2015).

Según el Informe Global Digital (Kemp, 2019) en el mundo está conectado el 57\% de los ciudadanos del planeta y ya más de la mitad de los cubanos se conectan a internet (6 470000 ), lo que representa el $56 \%$ de la población del país. Alertan las investigaciones que está emergiendo un nuevo tipo de desadaptaciones conductuales producto de la generalización de las TIC. Si bien estas herramientas fueron creadas como tecnologías para informar y comunicar, su propio diseño es susceptible de afectar a la voluntad de control, lo cual, junto con otros factores personales y ambientales, facilita la conducta adictiva.

El uso de las TIC requiere de un mayor esfuerzo, sobre todo si causan problemas o interfieren en la vida diaria, especialmente durante la adolescencia; una etapa que merece una atención personalizada pues transitan por la etapa más vulnerable del desarrollo humano. Los adolescentes, fascinados por internet, el móvil y los videojuegos, han encontrado en estas tecnologías un medio extraordinario de relación, comunicación, aprendizaje, satisfacción de la curiosidad, ocio y diversión (Rodríguez, 2019).

Por tanto, resulta necesario desplegar un organizado y planificado trabajo que fortalezca el proceso de orientación en este sentido. La orientación como relación de ayuda permite que los estudiantes puedan llegar a identificar sus propios patrones de riesgo, haciendo un uso saludable y responsable de la tecnología. Es fundamental 


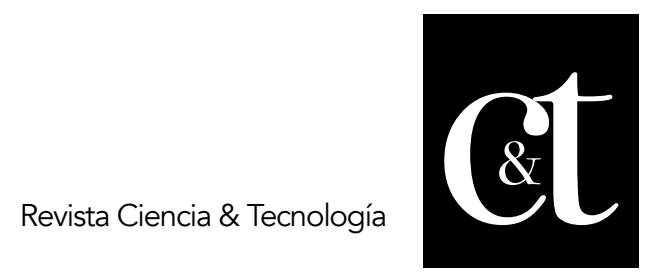

No. 28, 31 de octubre de 2020

ISSN impreso: 1390 - 6321

ISSN online: 2661 - 6734

orientar a los adolescentes para prevenir un uso inadecuado de las nuevas tecnologías, ya que de ser arraigada la dependencia tecnológica puede convertirse en una adicción.

Desde este punto de vista existen investigadores que han trabajado la orientación psicopedagógica y su relevancia en la prevención de diferentes situaciones que pueden presentarse en la etapa del desarrollo adolescente. Se destacan a nivel internacional Bisquerra (2009); Ordaz, Ramos y Pacheco (2018), quienes aportan sus consideraciones con respecto a las áreas, principios y modelos de intervención psicopedagógica en los diferentes contextos de interacción. A nivel nacional se parte de la definición vigotskiana de que la educación conduce el desarrollo (Vigotsky, 1988) y sobresalen los autores Collazo (2001), Rodríguez (2001), Calviño (2002), Del Pino y Recarey (2005); los cuales ofrecen definiciones de orientación, destacan sus clasificaciones de preventiva y desarrolladora y contribuyen con diferentes vías, métodos, técnicas y modalidades de orientación.

A nivel provincial se toma como referente los estudios de Rojas (2018), quien se refiere a la orientación psicopedagógica en el contexto educativo y ahonda además en la orientación profesional. Por su parte Vera (2020), propone una estrategia de orientación, pero en el nivel educativo Secundaria Básica y encaminada a la convivencia escolar.

A pesar de que existen autores que han trabajado lo relativo al uso de la tecnología y a la necesidad de realizar acciones encaminadas a prevenir la dependencia a ella por parte de los adolescentes, como Caicedo y Solano (2010); Holzmann (2016); Montañés (2017) en el ámbito mundial y Rodríguez (2019), en el ámbito nacional, aún resta profundizar en dicha temática desde el proceso de orientación psicopedagógica en el Preuniversitario, así como elaborar propuestas encaminadas a la prevención aprovechando las potencialidades que brindan las instituciones educativas y con la colaboración de las diversas influencias que reciben los estudiantes.

Es así que las investigaciones apuntan hacia la necesidad de prevenir la dependencia tecnológica en los adolescentes y que la orientación psicopedagógica juega un papel esencial en esta dirección, a pesar de que no son satisfechas todas las necesidades al respecto. Sin embargo, es posible constatar una contradicción entre tales intenciones y la realidad educativa, pues desde el proceso de orientación psicopedagógica existen deficiencias en las acciones que se realizan para la prevención de la dependencia tecnológica en los adolescentes. Es por ello que en el presente trabajo se propone una estrategia de orientación psicopedagógica para la prevención de la dependencia tecnológica en los adolescentes del Preuniversitario.

\section{Prevención de la dependencia tecnológica en adolescentes}

La orientación en el ámbito escolar es un proceso que se puede programar y dirigir, pero solo se realiza a través de la comunicación con el estudiante y estimulando su autodeterminación, protagonismo y responsabilidad con sus decisiones y conductas. Su esencia es ayudar al sujeto a conocerse y mejorarse a sí mismo, en relación con el medio en que se desenvuelve. Según Collazo (2001), orientar significa ayudar, guiar al individuo para que movilice conscientemente sus potencialidades, enfrente sus limitaciones y decida el curso de su vida. Para Vera (2020) la orientación es un proceso dinámico que incluye una serie de actividades que tienen por objetivo evaluar al individuo, estimularlo y "ayudarlo" para que logre desenvolverse con mayor independencia, autonomía e integración al grupo. Es así que Calviño (2002), considera que esta relación de ayuda es el vínculo interpersonal donde se movilizan, 


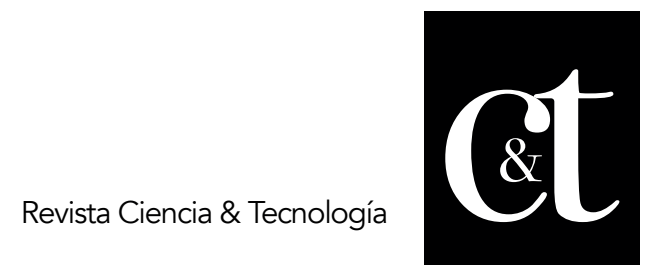

No. 28, 31 de octubre de 2020

ISSN impreso: 1390 - 6321

ISSN online: 2661 - 6734

en función del crecimiento personal y/o profesional, los recursos para una relación de ayuda profesional con un elemento esencial, facilitar el reconocimiento del problema para el que alguien necesita ayuda; es acompañarle en la búsqueda de alternativas para el enfrentamiento o solución del problema. Se considera que la orientación es una relación de ayuda profesional que se brinda a un sujeto o varios de ellos de forma planificada, preventiva, comprensiva, sistémica y desarrolladora, donde al orientador le corresponde apoyar, guiar, colaborar, conducir, estimular, propiciar el paso a niveles superiores de todo sujeto que requiera ser orientado y no por el contrario imponer criterios o soluciones, sobreproteger, abandonar o discriminar.

Se coincide con Rojas (2018), que adopta la concepción de la orientación psicopedagógica como el término más completo que en la actualidad puede abarcar a las diferentes denominaciones que se le han otorgado a la orientación. La concibe como el proceso de ayuda y acompañamiento continuo a todas las personas, en todos sus aspectos, con el propósito de potenciar la prevención y el desarrollo humano a lo largo de la vida, lo cual implica que la relación de ayuda se realiza mediante una intervención profesionalizada.

En las áreas a considerar en el proceso de orientación psicopedagógica se concibe según Rojas (2018), el área de la orientación profesional, la orientación en el proceso de enseñanza aprendizaje, la atención a la diversidad y como referencia para el estudio, aspectos que tributan al desarrollo del individuo y su integralidad, de ahí la existencia del área de orientación para la prevención y el desarrollo humano, que posee como propósito la prevención en el sentido amplio, así como el desarrollo personal y social. La orientación concibe entre sus principios además el principio del desarrollo, intervención social y de la prevención, que supone actuaciones de tipo proactivo; se trata de actuar antes de que surja el problema.

Según la Resolución 111/2017 en el Preuniversitario los consejos de escuela intervienen en la ejecución de las acciones diseñadas para el desarrollo de la labor preventiva en la institución educativa y exigen junto con el Consejo de dirección la labor educativa de los docentes y el apoyo de la familia en la formación de sus hijos. A su vez los psicopedagogos participan, asesoran y ejecutan, en coordinación con la dirección de la institución educativa, el proceso de implementación de la labor preventiva, priorizando en la caracterización individual, grupal y colectiva. De esta manera, en el Preuniversitario el proceso de orientación está encaminado a efectuar una relación de ayuda, que permita un desarrollo y crecimiento personal de los adolescentes y las demás agencias socializadoras, a partir del conocimiento de la realidad, así como acciones planificadas que permitan su ejecución y evaluación.

Es posible desde el proceso de orientación psicopedagógica en el Preuniversitario abordar temáticas complejas para la etapa del desarrollo adolescente tales como los proyectos de vida, las habilidades sociales, las relaciones interpersonales, las relativas a la salud y sexualidad, comunicación asertiva, autorregulación emocional o estimulación de hábitos de estudio favorables. Sin embargo, en la actualidad tienen lugar situaciones relativas al uso de la tecnología que requieren apoyo especializado desde un enfoque preventivo.

Para Rodríguez (2019) los adolescentes siempre han sido un grupo de riesgo en multitud de aspectos debido a los cambios que se dan en esta etapa; la tecnología se ha convertido en otro de ellos. La era digital ha cambiado la forma en la que se 


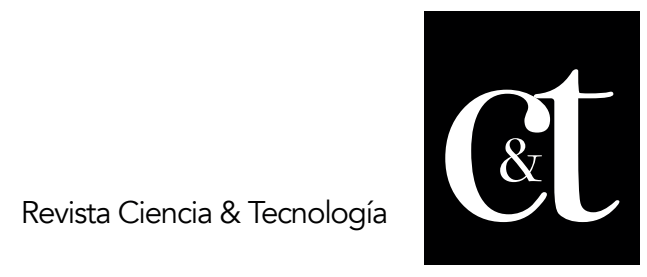

No. 28,31 de octubre de 2020

ISSN impreso: 1390 - 6321

ISSN online: 2661 - 6734

comunican y relacionan y han nacido nuevas formas de comportamientos no saludables en una multitud de partes que conforman la vida del adolescente.

Al tener en cuenta las concepciones anteriores se asume que en su desarrollo el adolescente transita por una serie de situaciones traumáticas, pues cuestiones que con anterioridad no representaban una problemática para ellos ahora tendrían que asumir una posición, ser responsable de sus actos, tomar decisiones. Un sinnúmero de dudas y preocupaciones se apoderan de ellos, tanto de los cambios que están ocurriendo en su cuerpo como las situaciones de su entorno, una pérdida, fracaso escolar, sobreprotección, separación de los padres, sentimientos de rechazo, son algunas de las cuestiones más comunes; el adolescente puede vivirlas en soledad en lugar de afrontarlas con sus padres, profesores, amigos.

En esa soledad, al decir de Encinas, Requesens y Helguera (2015), tiene la posibilidad de moverse en un espacio invisible ante los adultos y un mundo de fantasías, crear una identidad deseada y manifestarse no tal como realmente es sino como desearía ser o como los demás querrían que fuera. Puede sentirse superior ante un videojuego que controla, chatear a través de internet $\mathrm{y} / \mathrm{o}$ del teléfono móvil, crear un amplio grupo de amigos. Las TIC sin lugar a dudas se convierten en su mejor aliado y lo más extraordinario que les ha pasado en la vida, les va a dar la oportunidad de mostrarse perfecto ante el mundo y evadir todos los problemas que se desarrollen en su entorno.

El móvil interviene de manera significativa en la socialización porque permite definir la identidad del adolescente tanto individual, a base de personalizar el aparato de varias formas, colores, tonos, etc., como colectiva, creando un lenguaje especial de grupo, mensajes de texto y llamadas perdidas. Pero, aclara Rodríguez (2019), que al igual que se utiliza para establecer contactos a corta distancia, actúa así mismo de barrera de seguridad frente a los padres desde el momento en que estos no pueden acceder a su teléfono personal.

Pese a los múltiples beneficios del uso de la tecnología hay datos y estudios que alertan de los riesgos derivados de la masiva presencia de las nuevas tecnologías en la vida cotidiana de adolescentes. Sostiene Holzmann (2016), que una de las consecuencias más evidente de esa dedicación tan amplia a estas nuevas tecnologías es la disminución del tiempo dedicado a la realización de otras actividades necesarias y antes mucho más habituales, como leer, estudiar, salir a pasear, conversar personalmente o practicar deportes.

Las principales señales de alarma que denotan una dependencia a las tecnologías o a las redes sociales y que pueden ser un reflejo de la conversión de una afición en una adicción son las siguientes, a consideración de Holzmann (2016):

- Privarse de sueño (+5 horas) para estar conectado a la red, a la que se dedica unos tiempos de conexión anormalmente altos o interactuando con el móvil.

- Descuidar otras actividades importantes, como el contacto con la familia, las relaciones sociales, el estudio o el cuidado de la salud.

- Recibir quejas de alguien cercano, como los padres o los hermanos, en relación con el uso de la red o el móvil.

- Pensar en la red de manera constante, incluso cuando no se está conectado a ella y sentirse irritado de forma excesiva cuando la conexión falla o resulta muy lenta.

- Intentar limitar el tiempo de conexión o interacción con el móvil, pero sin conseguirlo y perder la noción del tiempo.

- Mentir sobre el tiempo real que se está conectado o jugando a un videojuego. 


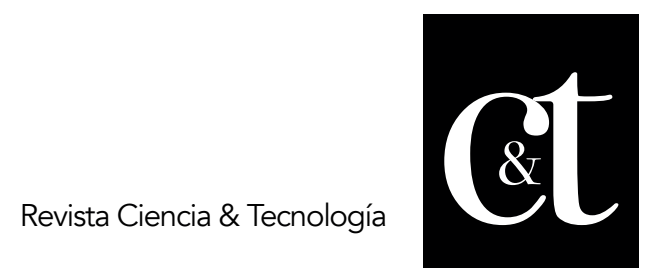

No. 28, 31 de octubre de 2020

ISSN impreso: 1390 - 6321

ISSN online: 2661 - 6734

- Aislarse socialmente, mostrarse irritable y bajar el rendimiento en los estudios.

- Sentir una euforia y activación anómalas cuando se está delante del móvil.

El uso desmedido e incontrolado de la tecnología genera dependencia y resta libertad al ser humano al estrechar su campo de conciencia y restringir la amplitud de sus intereses. Existen hábitos de conducta en apariencia inofensivos que, en determinadas circunstancias, pueden convertirse en adictivos e interferir en la vida cotidiana de las personas afectadas, a nivel familiar, escolar, social o de salud. Por eso, es importante diferenciar entre el uso frecuente de las nuevas tecnologías y la dependencia a las mismas, agrega Holzmann (2016).

Los autores Caicedo y Solano (2010), destacan que la dependencia tecnológica se puede entender como la urgencia de utilización de equipamientos o servicios tecnológicos que facilitan la ejecución de las actividades y la satisfacción de las necesidades de las personas en el desarrollo de sus tareas cotidianas. Refieren la dependencia como necesidad de desarrollar un comportamiento determinado por la satisfacción que produce el mismo, con la intención de eliminar el malestar que genera el no poder hacerlo. Por tanto, la dependencia tecnológica es que la persona tiene la necesidad compulsiva e incontrolada de estar sujeto a su dispositivo tecnológico como medio imprescindible en su vida.

Las causas de la dependencia tecnológica se originan en las limitaciones propias del ser humano y su deseo por satisfacer sus necesidades cada vez más crecientes, de esta manera se crea una espiral en la cual la satisfacción de las necesidades por medios tecnológicos crea nuevas necesidades y nuevos desarrollos tecnológicos para satisfacerlas, haciendo a la persona cada vez más dependiente. Indican Caicedo y Solano, (2010) que otras causas de dependencia tecnológica se originan en necesidades psicológicas insatisfechas, las cuales son aprovechadas por las llamadas nuevas tecnologías que encuentran en estos usuarios sus consumidores más compulsivos.

Además, se admite que lo que realmente representa un peligro no es tanto la frecuencia con que se realiza, sino la relación de dependencia que se crea, la pérdida de control sobre su conducta por parte del sujeto y la grave interferencia que genera en la vida cotidiana. En este sentido, no hay que confundir la dependencia del celular con sentirse atraído por las nuevas tecnologías, ni disfrutar de las redes sociales virtuales con ser adicto a Internet.

Datos a nivel mundial indican que el $50 \%$ de los adolescentes se considera adicto a la tecnología y el $48 \%$ de los que pasan más de cinco horas con su teléfono ha reportado sentimientos de depresión, aislamiento o suicidio (Alonso, 2018). El Centro de Estudios sobre la Juventud en Cuba desarrolló una investigación exploratoria a nivel nacional en el 2018 sobre el uso actual de las TIC por los adolescentes y jóvenes cubanos en sus prácticas sociales. El estudio se realizó con 1897 adolescentes y jóvenes de 12 a 34 años de edad de todo el país; el 45,0\% adolescentes y el 55,0\% jóvenes. Arrojó que en la semana el $83,9 \%$ de los adolescentes y jóvenes cubanos usa diariamente el teléfono móvil; el $59,9 \%$ define su nivel de manejo de tecnologías informáticas con la categoría alto. El $50 \%$ de los que la emplean son estudiantes y $20,5 \%$ están en el Preuniversitario. De ahí que se considera que el uso de la tecnología constituye un tema de suma importancia a tratar en la actualidad, por lo que es necesario la búsqueda de vías, alternativas, para prevenir que la dependencia a la tecnología se convierta en una amenaza o factor de riesgo para el adolescente.

Las herramientas tecnológicas son de gran importancia por lo que se debe fomentar su utilización entre los estudiantes, sin embargo, además de enseñarles cómo utilizar 


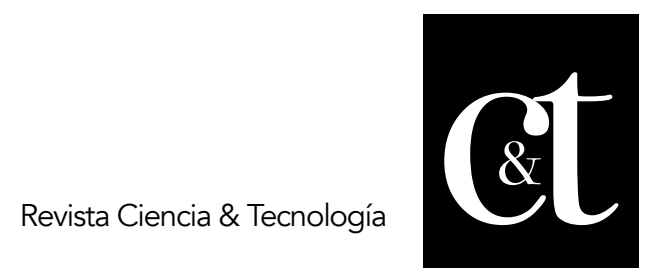

No. 28, 31 de octubre de 2020

ISSN impreso: 1390 - 6321

ISSN online: 2661 - 6734

la tecnología se les debe advertir acerca de los inconvenientes y las consecuencias que estas presentan. El maestro debe orientar al estudiante para que utilicen las herramientas tecnológicas como facilitadoras de tareas y que ayudan a mejorar las relaciones interpersonales. A los estudiantes se les debe dar libertad e incentivar para que investiguen y desarrollen habilidades en el uso de las nuevas tecnologías y aprendan a alternarla con periodos de descanso y recreación.

La prevención de la dependencia tecnológica en los adolescentes es concebida como un proceso encaminado a anticiparse y limitar en la medida de lo posible que las manifestaciones de dependencia tecnológica marquen de forma negativa el desarrollo de la personalidad de los adolescentes, mediante la estimulación del uso adecuado de la tecnología en los diferentes espacios de socialización. En este sentido, la orientación psicopedagógica se torna de gran relevancia, pues ofrece la posibilidad de concretar tales intenciones desde una relación de ayuda profesional y sistemática.

Se trata de que los adolescentes comprendan y aprendan alternativas adecuadas para el uso de la tecnología, sin que su contacto excesivo derive en importantes trastornos que afecten actividades básicas de estudio, socialización o recreación. Una propuesta de este tipo debe partir de la reflexión constante, el análisis de la realidad y las consecuencias a corto y largo plazo que se pueden enfrentar si no se realizan acciones de tipo proactivas. Además, es preciso lograr la participación e interacción de todos los implicados, de modo que surjan propuestas e iniciativas del debate realizado.

\section{Metodología}

En el presente estudio se tomó como población el 11 no grado (120 estudiantes) del Preuniversitario Martín Dihigo Llano del municipio Cienfuegos, que se encuentra formando parte del III Perfeccionamiento educacional en el país, lo que implica cambios en los programas que favorecen el uso de la tecnología como medio de desarrollo, actualización, adquisición y enriquecimiento de los conocimientos. Se obtuvo el consentimiento informado de los participantes, tanto estudiantes como docentes y directivos.

Como muestra para la investigación, de tipo intencional no probabilística, se seleccionó el grupo 11 no 2 con una matrícula de 28 estudiantes, de ellos 16 son hembras y 12 varones, con edades correspondientes entre los 16 y 17 años. A través de la observación se pudo identificar comportamientos desarrollados por los adolescentes en los horarios de receso y dentro del aula que pueden ser factores de riesgo para el uso desmedido e incontrolado de la tecnología.

Una vez seleccionado el grupo se aplicó una encuesta para identificar la frecuencia con la que desarrollan determinados comportamientos relacionados con el uso de la tecnología. A partir de estos resultados luego se efectuaron entrevistas a los estudiantes para comprobar la veracidad de la información ofrecida en la encuesta y otras cuestiones que no se encontraban inmersas en ella, como la implicación de los docentes en el proceso de orientar a los estudiantes sobre el uso de la tecnología (ventajas y desventajas) y utilización de ésta como medio de enseñanza en la docencia. Además, se entrevistó a docentes y directivos para recoger información e interpretar sus consideraciones respecto al uso de la tecnología, su implementación en la docencia y orientación para su adecuada utilización. Para enriquecer el diagnóstico se utilizó además el método de análisis de documentos, evidenciado en la revisión de la caracterización de la escuela y del grupo de muestra.

\section{Resultados}


A partir de la triangulación de los instrumentos aplicados se determinaron las siguientes potencialidades y necesidades.

Potencialidades:

- Reconocimiento de la problemática.

- Interés de los estudiantes por ser orientados sobre el adecuado uso de la tecnología.

- Disposición de los docentes y directivos para implementar acciones encaminadas a fomentar el proceso de orientación psicopedagógica en este sentido.

Necesidades:

- Manifestaciones que favorecen la dependencia tecnológica desarrollada por los estudiantes como el uso del móvil de manera excesiva, incluso en horarios de clase.

- Interpretación de la tecnología como distractor en la docencia por los docentes de mayor experiencia.

- Significativas brechas en el proceso de orientación psicopedagógica al no contemplar la prevención de la dependencia tecnológica como uno de sus contenidos.

De lo anterior es posible concluir que existen diversas situaciones que pueden conducir a los adolescentes del Preuniversitario a desarrollar una dependencia tecnológica que afecte su desarrollo armónico, de ahí la necesidad de perfeccionar la orientación psicopedagógica que tiene lugar y elaborar una Estrategia de orientación psicopedagógica que se constituya en una propuesta factible ante las necesidades detectadas.

Propuesta de Estrategia de orientación psicopedagógica para la prevención de la dependencia tecnológica en los adolescentes del Preuniversitario

Se decide asumir una estrategia de orientación psicopedagógica que ha sido objeto de trabajo de Vera (2020) y al decir de Rojas, et al., (2020), es la dirección de un sistema de acciones de orientación flexibles, sistémicas y sistemáticas, encaminadas al establecimiento de una relación de ayuda individual o grupal, que posibilite al orientado u orientados, transitar de un estado inicial y real de desarrollo, a un estado cualitativo superior, de modo que pueda resolver de manera consciente, independiente y reflexiva las tareas correspondientes a su edad y se promueva su evolución personal, social y/o profesional (p.50).

Los autores asumen que la estrategia de orientación constituye un sistema de objetivos dirigidos a potenciar, modificar, cambiar el desarrollo de un individuo o grupo, diseñada con los recursos de apoyo y ayuda que necesita cada individuo a partir de sus necesidades y potencialidades para alcanzar su máximo desarrollo.

Objetivo general de la estrategia: Contribuir al proceso de orientación psicopedagógica para la prevención de la dependencia tecnológica en los adolescentes del Preuniversitario.

La estrategia de orientación psicopedagógica está estructurada en cuatro etapas (diagnóstico, elaboración, ejecución y evaluación) que se corresponden con la estructura de la orientación sugerida por Del Pino y Recarey (2005). Se contemplan a la vez en la etapa de elaboración, cuatro macro acciones que contienen 13 actividades dirigidas a adolescentes, docentes, directivos y familiares. La estrategia se propone para ser implementada por un especialista de la orientación como el psicopedagogo que puede establecer niveles de ayuda y alternativas para prevenir la 
dependencia tecnológica con el apoyo de los métodos específicos de la orientación psicopedagógica.

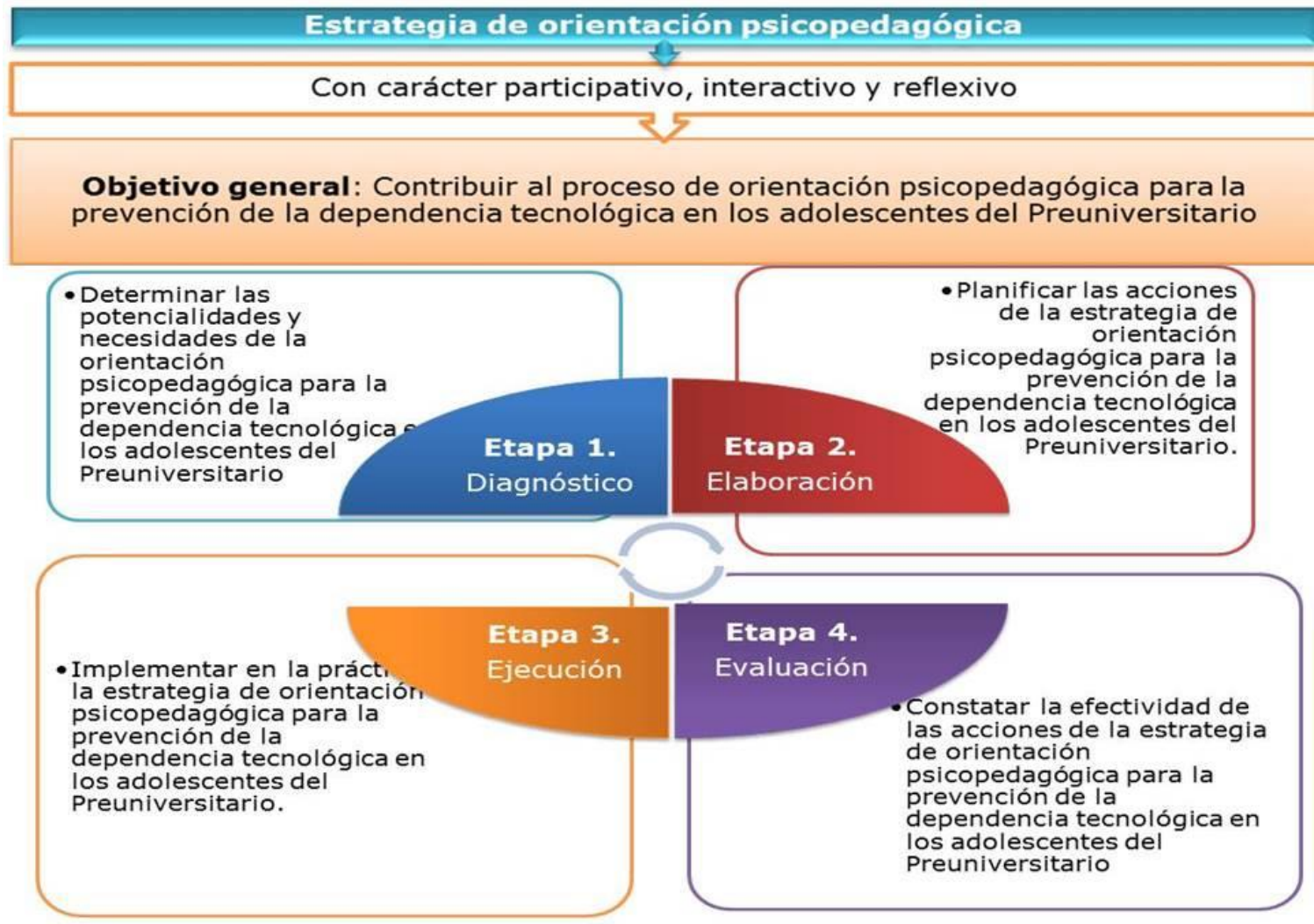

\section{Figura 1. Representación gráfica de la estrategia de orientación psicopedagógica}

Fuente: elaboración propia

Estas acciones de orientación se llevarán a cabo teniendo en cuenta los criterios de Collazo (2001), para su desarrollo exitoso:

1. Percepción, conciencia de la necesidad de ayuda; deseo y esperanza de cambio.

2. Establecimiento del rapport o relación emocional positiva psicológica y vencimiento de la resistencia, si existe.

3. Expresión y liberación de las emociones y sentimientos (catarsis).

4. Exploración más profunda de los sentimientos. Reflejar, (tratar de desentrañar y devolverle al individuo las mismas ideas, pero sin la carga emocional), clarificar (aclarar, ilustrar, poner un ejemplo para esclarecer al sujeto) y establecer hipótesis (acerca de la causa de la actitud que el sujeto expresa).

5. El proceso de abrir o desbrozar el camino (comprensión racional del problema).

6. Desarrollo de la comprensión de sí mismo o de la penetración y visión interna.

7. Experiencia vital fuera de los marcos de la relación con el orientador en particular o de la establecida dentro del grupo de orientación. (El sujeto debe experimentar en sus relaciones cotidianas un cambio favorable que refuerce y consolide las transformaciones ocurridas en su personalidad). 


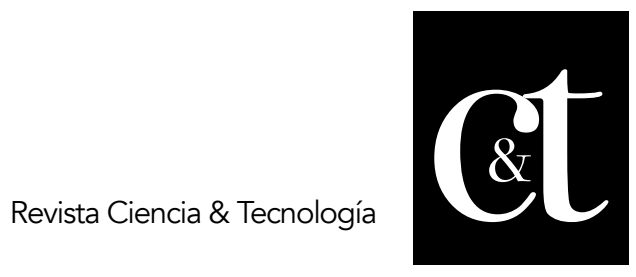

No. 28, 31 de octubre de 2020

ISSN impreso: 1390 - 6321

ISSN online: 2661 - 6734

El método fundamental del cual valerse para la orientación grupal es la discusión de grupo; que se basa en la aplicación de las etapas del pensamiento reflexivo para el análisis y discusión de los problemas con vistas a su solución. Además, se pueden emplear otras técnicas como el cine debate, la reflexión grupal, la entrevista de orientación, la solución de problemas y el reforzamiento.

La entrevista de orientación tiene la particularidad de que el objetivo fundamental de la misma es incidir de alguna manera en la otra persona para que, por ejemplo, modifique una actitud, abandone una conducta, eleve su autoestima en alguna dirección. Es decir, en este tipo de entrevista la incidencia es más importante que la obtención de información sobre el sujeto. No obstante, la entrevista mantendrá, como proceso, una dialéctica entre la información que se recibe del sujeto y la incidencia que se va logrando en él, ya que la primera permite nuevas interpretaciones de la situación y la modificación pertinente del mensaje.

La reflexión grupal consiste en la discusión de un tema de interés educativo, seleccionado a partir del diagnóstico del grupo (o que emerge a partir de una circunstancia dada) que se realiza en un grupo creado o utilizado para esos fines, permite que expresen sus dudas, sus puntos de vista y poder conocerlos mejor.

La solución de problemas se encamina a la modelación, a través de la colaboración con el estudiante, del proceso de análisis, síntesis y toma de decisión ante un problema determinado. En esta técnica se ayuda al estudiante a ordenar sus ideas, (que pueden estar bloqueadas por un estado emocional especial) y llegar a una solución desarrolladora del problema que enfrenta.

El reforzamiento hace referencia a una acción que hace al estudiante tomar conciencia de una cualidad, un éxito, una posibilidad que posea; se refuerza un aspecto positivo.

En relación a la composición de la estrategia, la acción de familiarización y sensibilización de los implicados con la temática incluye entrevista de orientación grupal, taller docente y taller de orientación a padres. Con posterioridad para la identificación de comportamientos que genera el uso de la tecnología se concibe el cine-debate, rol-playing, taller docente y taller de orientación a padres. La autoevaluación de comportamientos y la proyección de las transformaciones parten de la reflexión grupal, el reforzamiento, la solución de problemas y la entrevista de orientación grupal. Así mismo, la dinámica grupal y el taller permiten evaluar resultados de las acciones de orientación implementadas.

Rasgos que caracterizan a la estrategia de orientación psicopedagógica La estrategia de orientación psicopedagógica tiene un carácter participativo, interactivo y reflexivo para estimular el uso adecuado de la tecnología a modo de contribuir a la prevención de la dependencia tecnológica en los adolescentes del Preuniversitario.

Carácter participativo: resulta una estrategia que prioriza y fomenta la participación activa y protagónica de los adolescentes, familiares y docentes, transitando por la concepción de las etapas que conforman la estrategia hasta su ejecución y evaluación.

Carácter interactivo: resulta una característica fundamental de la estrategia de orientación psicopedagógica el trabajo en grupo, facilitando la interacción entre los participantes a modo de mejorar sus habilidades sociales y el aprendizaje mutuo a 


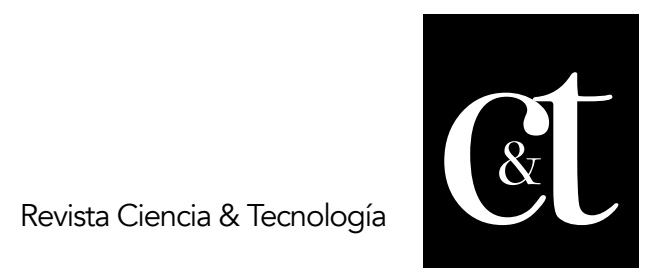

No. 28,31 de octubre de 2020

ISSN impreso: 1390 - 6321

ISSN online: 2661 - 6734

partir de las experiencias y criterios del resto de los compañeros. Predomina el uso de dinámicas y técnicas grupales que respalden la interacción entre los miembros.

Carácter reflexivo: se acentúa el valor de la reflexión intencionada y sistemática, el análisis y diálogo sobre la realidad educativa para la prevención de la dependencia tecnológica en los adolescentes, que se involucran en un proceso interactivo de cambio y desarrollo, lo que implica autorregulación, autoconocimiento, responsabilidad y creatividad.

Requisitos para la implementación de la estrategia de orientación psicopedagógica:

- Aseguramiento de las condiciones para el desarrollo de las actividades de la estrategia de orientación psicopedagógica (disponibilidad de locales, aseguramiento material, planificación de horario).

- Convocar a los adolescentes, familiares y docentes para garantizar la participación en las actividades de la estrategia de orientación psicopedagógica.

- Preparación de los medios y materiales que se utilizarán en las actividades planificadas.

\section{Resultados preliminares del proceso de implementación de la propuesta}

En sentido general predominaron en las actividades la utilización de técnicas proyectivas, participativas y grupales; así como la utilización de medios de enseñanza, lo que facilitó el desarrollo de las actividades de una forma dinámica, atractiva y provechosa para un mejor entendimiento, interés y motivación en los participantes.

La participación de los adolescentes fue buena, con un $92.3 \%$ de asistencia como promedio, con los padres el $76.5 \%$ y con los docentes se logró el $95 \%$. De manera general mostraron interés por la temática en cuestión y expresaron criterios coherentes y en correspondencia con sus vivencias. Resaltaron el papel activo de los métodos empleados y lo atractivo de los medios y materiales, predominaron las actividades de carácter grupal, que exigieron la interacción y el debate y recibieron los niveles de ayuda solicitados. A partir del procesamiento de los resultados de la entrevista a los participantes (adolescentes, familiares, docentes) para comprobar nivel de satisfacción con la estrategia y el registro sistemático de observación de las acciones implementadas, se establecen como resultados preliminares derivados de la implementación de la propuesta las siguientes:

- Los adolescentes reflexionaron en relación a que no se trata de abandonar la tecnología, si no de utilizarla de manera adecuada para que no interfiera en su desarrollo personal, académico, familiar y social.

- Se concientizó la necesidad de orientar, facilitar y apoyar a los adolescentes, sobre todo en relación a temáticas actuales, como el uso de la tecnología y la prevención de su dependencia.

- El diálogo y la participación constituyeron un estímulo y fuente indispensable de saberes para la mejora educacional, surgieron ideas sobre lo que se hacía, cómo se hacía y qué podían transformar para perfeccionar el proceso de orientación psicopedagógica en temáticas como el uso de la tecnología.

- Se demostró cómo la concepción de propuestas desde el proceso de orientación psicopedagógica puede contribuir a estrechar los vínculos entre las influencias educativas y desarrollar acciones con carácter preventivo ante situaciones complejas de la etapa del desarrollo adolescente. 


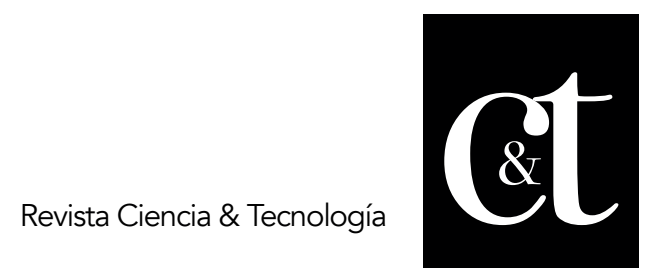

No. 28,31 de octubre de 2020

ISSN impreso: 1390 - 6321

ISSN online: 2661 - 6734

- Los docentes comprendieron la necesidad de emplear la tecnología como medio de enseñanza en las clases y en especial permitir que el estudiante se apoye en el móvil para actividades docentes.

- Se incentivó la autogestión de la información sobre la base de sus necesidades.

\section{Conclusiones}

En el proceso de orientación psicopedagógica en el Preuniversitario se deben abordar temáticas complejas relativas a la etapa del desarrollo adolescente. En este caso las nuevas tecnologías se han convertido en un factor de riesgo para los adolescentes que las emplean de manera desmedida e incontrolada, llegando a convertirse en una dependencia, por lo que se precisa contribuir desde la orientación psicopedagógica a la prevención de la dependencia tecnológica en los adolescentes del Preuniversitario.

Los resultados obtenidos en el estudio empírico a partir de los instrumentos aplicados en el Preuniversitario Martín Dihigo Llano del municipio Cienfuegos evidenciaron significativas brechas en el proceso de orientación psicopedagógica en relación al uso adecuado de la tecnología, cuestión que ha posibilitado la identificación de rasgos dependientes a la tecnología en los adolescentes y el empleo deficiente de ésta en las actividades docentes. En el diseño de una estrategia de orientación psicopedagógica para la prevención de la dependencia tecnológica en los adolescentes del Preuniversitario se tuvieron en cuenta las potencialidades y necesidades del proceso de orientación en este sentido. La estrategia se organiza en cuatro etapas que se corresponden con la estructura de la orientación, posee un carácter reflexivo, participativo e interactivo, estimula el uso adecuado de la tecnología e involucra a adolescentes, docentes y familiares.

La implementación de la estrategia culminó con resultados satisfactorios, los adolescentes se mostraron interesados, reflexivos y con una participación activa. Los docentes refirieron sobre los beneficios de la utilización de la tecnología en las clases, así como la necesidad de orientar a los adolescentes sobre el uso adecuado de la tecnología. Los familiares manifestaron la disposición de continuar profundizando y sistematizando acerca de la temática. Se perciben modificaciones en el proceso de orientación psicopedagógica que se desarrolla en el Preuniversitario para la prevención de la dependencia tecnológica en los adolescentes.

\section{Referencias bibliográficas}

Alonso, N. (12 de febrero de 2018). La tecnología es un producto adictivo y debe ser regulado como tal. Washington. http://tecnología/1518170231_523757.html

Bisquerra, A. R. (2009). La orientación como proceso educativo. Madrid: Morata.

Caicedo Suarez, U., y Solano Campo, H. (2010). Dependencia tecnológica. (Diplomado Docencia Universitaria). Universidad de la Guajira.

Calviño, M. (2002). Orientación psicológica. Esquema referencial de alternativas múltiples. La Habana: Científico Técnica.

Collazo Delgado, B. (2001). La orientación en la actividad pedagógica. La Habana: Pueblo y Educación.

Del Pino Calderón, J. L., y Recarey Fernández, S. (2005). La orientación educacional y la facilitación del desarrollo desde el rol profesional del maestro. La Habana:Unesco. 


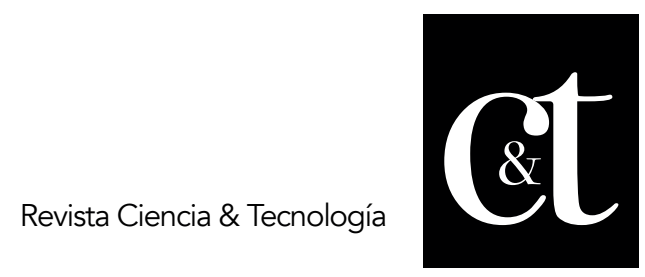

No. 28,31 de octubre de 2020

ISSN impreso: 1390 - 6321

ISSN online: 2661 - 6734

Encinas, F. I., Requesens, A. M., y Helguera Fuentes, M. (2015). Guía para padres y educadores sobre el uso seguro de Internet, móviles y videojuegos. Madrid: Fundación Gaudium.

Holzmann, A (2016). Adicción a la tecnología ¿qué es y cómo prevenirla? https://usuaria.org.ar/2017/10/12/adiccion-a-la-tecnologia-que-es-y-comoprevenirla/

Juventud Rebelde (2018, 7 de marzo). Juventud y tecnologías. Breve panorámica de los adolescentes y jóvenes y el uso de las TIC.

Kemp, S. (2019). Digital 2019 essential insights into how people around the world use the internet, mobile devices, social media, and e-commerce. Hootsuite. https://www.juancmejia.com/wp-content/uploads/2019/03/Digital-2019WeAreSocial-y-HootSuite.pdf

Ministerio de Educación de Cuba. (2017). Resolución 111/2017. La Habana: Pueblo y Educación.

Ministerio de Educación de Cuba. (2017). Plan de estudio provisional. La Habana:Pueblo y Educación.

Montañés, M. C. (2017). Prevención de las adicciones tecnológicas en la adolescencia. Padres y maestros, (369), 53-59.

Ordaz, M., Ramos, R., Pacheco, V. (2018). Proceso de orientación psicopedagógica sobre diversidad sexual en los estudiantes universitarios. Propuesta teórica para su implementación en los contextos de la Educación Superior Cubana. Investigación, socialización y desarrollo, 4 (61).

Rodríguez, E.S. (2001). Prospectiva del diagnóstico y la orientación. https://core. ac.uk/download/pdf/78633733.pdf

Rodríguez, J.M. (2019). Consejos para prevenir la adicción a la tecnología también en vacaciones. https://www.educaciontrespuntocero.com.html

Rojas Valladares, A. L. (2018). La orientación psicopedagógica en el contexto educativo. Cienfuegos: Universo Sur.

Rojas Valladares, A. L., Domínguez Urdanivia, Y., Torres Zerquera, L. C., y Pérez Egües, M. A. (2020). El proceso de intervención psicopedagógica en el ámbito educativo. Revista Metropolitana de Ciencias Aplicadas, 3(2), 45-51.

Vera, Y. (2020). La orientación educativa para la convivencia escolar de los adolescentes de Secundaria Básica. Tesis de Maestría, Universidad de Cienfuegos.

Vigotski, L. (1988). Interacción entre enseñanza y desarrollo. Selección de Lecturas de Psicología de las Edades I, Tomo III, Facultad de Psicología, Universidad de La Habana. 